\title{
An eigenvalue localization set for tensors and its applications
}

Jianxing Zhao* (D) and Caili Sang

${ }^{*}$ Correspondence: zjx810204@163.com

College of Data Science and

Information Engineering, Guizhou

Minzu University, Guiyang, Guizhou

550025, P.R. China

\begin{abstract}
A new eigenvalue localization set for tensors is given and proved to be tighter than those presented by Li et al. (Linear Algebra Appl. 481:36-53, 2015) and Huang et al. (J. Inequal. Appl. 2016:254, 2016). As an application of this set, new bounds for the minimum eigenvalue of $\mathcal{M}$-tensors are established and proved to be sharper than some known results. Compared with the results obtained by Huang et al., the advantage of our results is that, without considering the selection of nonempty proper subsets $S$ of $N=\{1,2, \ldots, n\}$, we can obtain a tighter eigenvalue localization set for tensors and sharper bounds for the minimum eigenvalue of $\mathcal{M}$-tensors. Finally, numerical examples are given to verify the theoretical results.
\end{abstract}

MSC: 15A18; 15A69; 65F10; 65F15

Keywords: $\mathcal{M}$-tensors; nonnegative tensors; minimum eigenvalue; localization set

\section{Introduction}

For a positive integer $n, n \geq 2, N$ denotes the set $\{1,2, \ldots, n\}$. $\mathbb{C}$ (respectively, $\mathbb{R}$ ) denotes the set of all complex (respectively, real) numbers. We call $\mathcal{A}=\left(a_{i_{1} \cdots i_{m}}\right)$ a complex (real) tensor of order $m$ dimension $n$, denoted by $\mathbb{C}^{[m, n]}\left(\mathbb{R}^{[m, n]}\right)$, if

$$
a_{i_{1} \cdots i_{m}} \in \mathbb{C}(\mathbb{R})
$$

where $i_{j} \in N$ for $j=1,2, \ldots, m$. $\mathcal{A}$ is called reducible if there exists a nonempty proper index subset $\mathbb{I} \subset N$ such that

$$
a_{i_{1} i_{2} \cdots i_{m}}=0, \quad \forall i_{1} \in \mathbb{J}, \forall i_{2}, \ldots, i_{m} \notin \mathbb{J} .
$$

If $\mathcal{A}$ is not reducible, then we call $\mathcal{A}$ irreducible [3].

Given a tensor $\mathcal{A}=\left(a_{i_{1} \cdots i_{m}}\right) \in \mathbb{C}^{[m, n]}$, if there are $\lambda \in \mathbb{C}$ and $x=\left(x_{1}, x_{2}, \ldots, x_{n}\right)^{T} \in \mathbb{C} \backslash\{0\}$ such that

$$
\mathcal{A} x^{m-1}=\lambda x^{[m-1]},
$$

(c) The Author(s) 2017. This article is distributed under the terms of the Creative Commons Attribution 4.0 International License (http://creativecommons.org/licenses/by/4.0/), which permits unrestricted use, distribution, and reproduction in any medium, provided you give appropriate credit to the original author(s) and the source, provide a link to the Creative Commons license, and indicate if changes were made. 
then $\lambda$ is called an eigenvalue of $\mathcal{A}$ and $x$ an eigenvector of $\mathcal{A}$ associated with $\lambda$, where $\mathcal{A} x^{m-1}$ is an $n$ dimension vector whose $i$ th component is

$$
\left(\mathcal{A} x^{m-1}\right)_{i}=\sum_{i_{2}, \ldots, i_{m} \in N} a_{i i_{2} \cdots i_{m}} x_{i_{2}} \cdots x_{i_{m}}
$$

and

$$
x^{[m-1]}=\left(x_{1}^{m-1}, x_{2}^{m-1}, \ldots, x_{n}^{m-1}\right)^{T} .
$$

If $\lambda$ and $x$ are all real, then $\lambda$ is called an $H$-eigenvalue of $\mathcal{A}$ and $x$ an $H$-eigenvector of $\mathcal{A}$ associated with $\lambda$; see $[4,5]$. Moreover, the spectral radius $\rho(\mathcal{A})$ of $\mathcal{A}$ is defined as

$$
\rho(\mathcal{A})=\max \{|\lambda|: \lambda \in \sigma(\mathcal{A})\},
$$

where $\sigma(\mathcal{A})$ is the spectrum of $\mathcal{A}$, that is, $\sigma(\mathcal{A})=\{\lambda: \lambda$ is an eigenvalue of $\mathcal{A}\}$; see $[3,6]$.

A real tensor $\mathcal{A}$ is called an $\mathcal{M}$-tensor if there exist a nonnegative tensor $\mathcal{B}$ and a positive number $\alpha>\rho(\mathcal{B})$ such that $\mathcal{A}=\alpha \mathcal{I}-\mathcal{B}$, where $\mathcal{I}$ is called the unit tensor with its entries

$$
\delta_{i_{1} \cdots i_{m}}= \begin{cases}1 & \text { if } i_{1}=\cdots=i_{m} \\ 0 & \text { otherwise }\end{cases}
$$

Denote by $\tau(\mathcal{A})$ the minimal value of the real part of all eigenvalues of an $\mathcal{M}$-tensor $\mathcal{A}$. Then $\tau(\mathcal{A})>0$ is an eigenvalue of $\mathcal{A}$ with a nonnegative eigenvector. If $\mathcal{A}$ is irreducible, then $\tau(\mathcal{A})$ is the unique eigenvalue with a positive eigenvector [7-9].

Recently, many people have focused on locating eigenvalues of tensors and using obtained eigenvalue inclusion theorems to determine the positive definiteness of an evenorder real symmetric tensor or to give the lower and upper bounds for the spectral radius of nonnegative tensors and the minimum eigenvalue of $\mathcal{M}$-tensors. For details, see $[1,2$, $10-14]$.

In 2015, Li et al. [1] proposed the following Brauer-type eigenvalue localization set for tensors.

Theorem 1 ([1], Theorem 6) Let $\mathcal{A}=\left(a_{i_{1} \cdots i_{m}}\right) \in \mathbb{C}^{[m, n]}$. Then

$$
\sigma(\mathcal{A}) \subseteq \Delta(\mathcal{A})=\bigcup_{i, j \in N, j \neq i} \Delta_{i}^{j}(\mathcal{A})
$$

where

$$
\begin{aligned}
& \Delta_{i}^{j}(\mathcal{A})=\left\{z \in \mathbb{C}:\left|\left(z-a_{i \cdots i}\right)\left(z-a_{j \cdots j}\right)-a_{i j \cdots j} a_{j i \cdots i}\right| \leq\left|z-a_{j \cdots j}\right| r_{i}^{j}(\mathcal{A})+\left|a_{i j \cdots j}\right| r_{j}^{i}(\mathcal{A})\right\}, \\
& r_{i}(\mathcal{A})=\sum_{\delta_{i i_{2} \cdots i_{m}}=0}\left|a_{i i_{2} \cdots i_{m}}\right|, \quad r_{i}^{j}(\mathcal{A})=\sum_{\substack{\delta_{i i_{2} \cdots i_{m}}=0, \delta_{j i_{2} \cdots i_{m}}=0}}\left|a_{i i_{2} \cdots i_{m}}\right|=r_{i}(\mathcal{A})-\left|a_{i j \ldots j}\right| .
\end{aligned}
$$

To reduce computations, Huang et al. [2] presented an $S$-type eigenvalue localization set by breaking $N$ into disjoint subsets $S$ and $\bar{S}$, where $\bar{S}$ is the complement of $S$ in $N$. 
Theorem 2 ([2], Theorem 3.1) Let $\mathcal{A}=\left(a_{i_{1} \cdots i_{m}}\right) \in \mathbb{C}^{[m, n]}, S$ be a nonempty proper subset of $N, \bar{S}$ be the complement of $S$ in $N$. Then

$$
\sigma(\mathcal{A}) \subseteq \Delta^{S}(\mathcal{A})=\left(\bigcup_{i \in S, j \in \bar{S}} \Delta_{i}^{j}(\mathcal{A})\right) \cup\left(\bigcup_{i \in \bar{S}, j \in S} \Delta_{i}^{j}(\mathcal{A})\right)
$$

Based on Theorem 2, Huang et al. [2] obtained the following lower and upper bounds for the minimum eigenvalue of $\mathcal{M}$-tensors.

Theorem 3 ([2], Theorem 3.6) Let $\mathcal{A}=\left(a_{i_{1} \cdots i_{m}}\right) \in \mathbb{R}^{[m, n]}$ be an $\mathcal{M}$-tensor, $S$ be a nonempty proper subset of $N, \bar{S}$ be the complement of $S$ in $N$. Then

$$
\min \left\{\min _{i \in S} \max _{j \in \bar{S}} L_{i j}(\mathcal{A}), \min _{i \in \bar{S}} \max _{j \in S} L_{i j}(\mathcal{A})\right\} \leq \tau(\mathcal{A}) \leq \max \left\{\max _{i \in S} \min _{j \in \bar{S}} L_{i j}(\mathcal{A}), \max _{i \in \bar{S}} \min _{j \in S} L_{i j}(\mathcal{A})\right\},
$$

where

$$
L_{i j}(\mathcal{A})=\frac{1}{2}\left\{a_{i \cdots i}+a_{j \ldots j}-r_{i}^{j}(\mathcal{A})-\left[\left(a_{i \cdots i}-a_{j \cdots j}-r_{i}^{j}(\mathcal{A})\right)^{2}-4 a_{i j \ldots j} r_{j}(\mathcal{A})\right]^{\frac{1}{2}}\right\} .
$$

The main aim of this paper is to give a new eigenvalue inclusion set for tensors and prove that this set is tighter than those in Theorems 1 and 2 without considering the selection of $S$. And then we use this set to obtain new lower and upper bounds for the minimum eigenvalue of $\mathcal{M}$-tensors and prove that new bounds are sharper than those in Theorem 3.

\section{Main results}

Now, we give a new eigenvalue inclusion set for tensors and establish the comparison between this set with those in Theorems 1 and 2 .

Theorem 4 Let $\mathcal{A}=\left(a_{i_{1} \cdots i_{m}}\right) \in \mathbb{C}^{[m, n]}$. Then

$$
\sigma(\mathcal{A}) \subseteq \Delta^{\cap}(\mathcal{A})=\bigcup_{i \in N} \bigcap_{j \in N, j \neq i} \Delta_{i}^{j}(\mathcal{A})
$$

Proof For any $\lambda \in \sigma(\mathcal{A})$, let $x=\left(x_{1}, \ldots, x_{n}\right)^{T} \in \mathbb{C}^{n} \backslash\{0\}$ be an associated eigenvector, i.e.,

$$
\mathcal{A} x^{m-1}=\lambda x^{[m-1]} \text {. }
$$

Let $\left|x_{p}\right|=\max \left\{\left|x_{i}\right|: i \in N\right\}$. Then $\left|x_{p}\right|>0$. For any $j \in N, j \neq p$, then from (1) we have

$$
\lambda x_{p}^{m-1}=\sum_{\substack{\delta_{p i_{2} \cdots i_{m}}=0, \delta_{j i_{2} \cdots i_{m}}=0}} a_{p i_{2} \cdots i_{m}} x_{i_{2}} \cdots x_{i_{m}}+a_{p \cdots p} x_{p}^{m-1}+a_{p j \cdots j} x_{j}^{m-1}
$$

and

$$
\lambda x_{j}^{m-1}=\sum_{\substack{\delta_{j i_{2} \cdots i_{m}}=0, \delta_{p i_{2} \cdots i_{m}}=0}} a_{j i_{2} \cdots i_{m}} x_{i_{2}} \cdots x_{i_{m}}+a_{j \cdots j} x_{j}^{m-1}+a_{j p \cdots p} x_{p}^{m-1},
$$


equivalently,

$$
\left(\lambda-a_{p \cdots p}\right) x_{p}^{m-1}-a_{p j \cdots j} x_{j}^{m-1}=\sum_{\substack{\delta_{p i_{2} \cdots i_{m}=0,} \\ \delta_{i_{2} \cdots i_{m}}=0}} a_{p i_{2} \cdots i_{m}} x_{i_{2}} \cdots x_{i_{m}}
$$

and

$$
\left(\lambda-a_{j \cdots j}\right) x_{j}^{m-1}-a_{j p \cdots p} x_{p}^{m-1}=\sum_{\substack{\delta_{j i_{2} \cdots i_{m}}=0, \delta_{p i_{2} \cdots i_{m}}=0}} a_{j i_{2} \cdots i_{m}} x_{i_{2}} \cdots x_{i_{m}} .
$$

Solving $x_{p}^{m-1}$ from (2) and (3), we get

$$
\begin{aligned}
& \left(\left(\lambda-a_{p \cdots p}\right)\left(\lambda-a_{j \cdots j}\right)-a_{p j \cdots j} a_{j p \cdots p}\right) x_{p}^{m-1} \\
& \quad=\left(\lambda-a_{j \cdots j}\right) \sum_{\substack{\delta_{p i_{2} \cdots i_{m}=0,}=0 \\
\delta_{j_{2} \cdots i_{m}}=0}} a_{p i_{2} \cdots i_{m}} x_{i_{2}} \cdots x_{i_{m}}+a_{p j \cdots j} \sum_{\substack{\delta_{j i_{2} \cdots i_{m}}=0, \delta_{p i_{2} \cdots i_{m}}=0}} a_{j i_{2} \cdots i_{m}} x_{i_{2}} \cdots x_{i_{m}} .
\end{aligned}
$$

Taking absolute values and using the triangle inequality yields

$$
\begin{aligned}
\mid(\lambda & \left.-a_{p \ldots p}\right)\left(\lambda-a_{j \ldots j}\right)-\left.a_{p j \ldots j} a_{j p \ldots p}|| x_{p}\right|^{m-1} \\
\quad & \leq\left|\lambda-a_{j \ldots j}\right| r_{p}^{j}(\mathcal{A})\left|x_{p}\right|^{m-1}+\left|a_{p j \ldots j}\right| r_{j}^{p}(\mathcal{A})\left|x_{p}\right|^{m-1} .
\end{aligned}
$$

Furthermore, by $\left|x_{p}\right|>0$, we have

$$
\left|\left(\lambda-a_{p \cdots p}\right)\left(\lambda-a_{j \ldots j}\right)-a_{p j \ldots j} a_{j p \cdots p}\right| \leq\left|\lambda-a_{j \ldots j}\right| r_{p}^{j}(\mathcal{A})+\left|a_{p j \ldots j}\right| r_{j}^{p}(\mathcal{A}),
$$

which implies that $\lambda \in \Delta_{p}^{j}(\mathcal{A})$. From the arbitrariness of $j$, we have $\lambda \in \bigcap_{j \in N, j \neq p} \Delta_{p}^{j}(\mathcal{A})$. Furthermore, we have $\lambda \in \bigcup_{i \in N} \bigcap_{j \in N, j \neq i} \Delta_{i}^{j}(\mathcal{A})$. The conclusion follows.

Next, a comparison theorem is given for Theorems 1, 2 and 4.

Theorem 5 Let $\mathcal{A}=\left(a_{i_{1} \cdots i_{m}}\right) \in \mathbb{C}^{[m, n]}, S$ be a nonempty proper subset of $N$. Then

$$
\Delta^{\cap}(\mathcal{A}) \subseteq \Delta^{S}(\mathcal{A}) \subseteq \Delta(\mathcal{A})
$$

Proof By Theorem 3.2 in [2], $\Delta^{S}(\mathcal{A}) \subseteq \Delta(\mathcal{A})$. Here, only $\Delta^{\cap}(\mathcal{A}) \subseteq \Delta^{S}(\mathcal{A})$ is proved. Let $z \in \Delta^{\cap}(\mathcal{A})$, then there exists some $i_{0} \in N$ such that $z \in \Delta_{i_{0}}^{j}(\mathcal{A}), \forall j \in N, j \neq i_{0}$. Let $\bar{S}$ be the complement of $S$ in $N$. If $i_{0} \in S$, then taking $j \in \bar{S}$, obviously, $z \in \bigcup_{i_{0} \in S, j \in \bar{S}} \Delta_{i_{0}}^{j}(\mathcal{A}) \subseteq$ $\Delta^{S}(\mathcal{A})$. If $i_{0} \in \bar{S}$, then taking $j \in S$, obviously, $z \in \bigcup_{i_{0} \in \bar{S}, j \in S} \Delta_{i_{0}}^{j}(\mathcal{A}) \subseteq \Delta^{S}(\mathcal{A})$. The conclusion follows.

Remark 1 Theorem 5 shows that the set $\Delta^{n}(\mathcal{A})$ in Theorem 4 is tighter than those in Theorems 1 and 2, that is, $\Delta^{\cap}(\mathcal{A})$ can capture all eigenvalues of $\mathcal{A}$ more precisely than $\Delta(\mathcal{A})$ and $\Delta^{S}(\mathcal{A})$. 
In the following, we give new lower and upper bounds for the minimum eigenvalue of $\mathcal{M}$-tensors.

Theorem 6 Let $\mathcal{A}=\left(a_{i_{1} \cdots i_{m}}\right) \in \mathbb{R}^{[m, n]}$ be an irreducible $\mathcal{M}$-tensor. Then

$$
\min _{i \in N} \max _{j \neq i} L_{i j}(\mathcal{A}) \leq \tau(\mathcal{A}) \leq \max _{i \in N} \min _{j \neq i} L_{i j}(\mathcal{A}) .
$$

Proof Let $x=\left(x_{1}, x_{2}, \ldots, x_{n}\right)^{T}$ be an associated positive eigenvector of $\mathcal{A}$ corresponding to $\tau(\mathcal{A})$, i.e.,

$$
\mathcal{A} x^{m-1}=\tau(\mathcal{A}) x^{[m-1]} .
$$

(I) Let $x_{q}=\min \left\{x_{i}: i \in N\right\}$. For any $j \in N, j \neq q$, we have by (4) that

$$
\tau(\mathcal{A}) x_{q}^{m-1}=\sum_{\substack{\delta_{q i_{2} \cdots i_{m}=0,} \\ \delta_{j i_{2} \cdots i_{m}}=0}} a_{q i_{2} \cdots i_{m}} x_{i_{2}} \cdots x_{i_{m}}+a_{q \cdots q} x_{q}^{m-1}+a_{q j \cdots j} x_{j}^{m-1}
$$

and

$$
\tau(\mathcal{A}) x_{j}^{m-1}=\sum_{\substack{\delta_{j i_{2} \cdots i_{m}=0}=0 \\ \delta_{q i_{2} \cdots i_{m}}=0}} a_{j i_{2} \cdots i_{m}} x_{i_{2}} \cdots x_{i_{m}}+a_{j \cdots j} x_{j}^{m-1}+a_{j q \cdots q} x_{q}^{m-1},
$$

equivalently,

$$
\left(\tau(\mathcal{A})-a_{q \cdots q}\right) x_{q}^{m-1}-a_{q j \cdots j} x_{j}^{m-1}=\sum_{\substack{\delta_{q i_{2} \cdots i_{m}}=0, \delta_{j i_{2} \cdots i_{m}}=0}} a_{q i_{2} \cdots i_{m}} x_{i_{2}} \cdots x_{i_{m}}
$$

and

$$
\left(\tau(\mathcal{A})-a_{j \cdots j}\right) x_{j}^{m-1}-a_{j q \cdots q} x_{q}^{m-1}=\sum_{\substack{\delta_{j i_{2} \cdots i_{m}}=0, \delta_{q i_{2} \cdots i_{m}}=0}} a_{j i_{2} \cdots i_{m}} x_{i_{2}} \cdots x_{i_{m}} .
$$

Solving $x_{q}^{m-1}$ by (5) and (6), we get

$$
\begin{aligned}
& \left(\left(\tau(\mathcal{A})-a_{q \cdots q}\right)\left(\tau(\mathcal{A})-a_{j \cdots j}\right)-a_{q j \cdots j} a_{j q \cdots q}\right) x_{q}^{m-1} \\
& \quad=\left(\tau(\mathcal{A})-a_{j \cdots j}\right) \sum_{\substack{\delta_{q i_{2} \cdots i_{m}}=0, \delta_{j_{2} \cdots i_{m}}=0}} a_{q i_{2} \cdots i_{m}} x_{i_{2}} \cdots x_{i_{m}}+a_{q j \cdots j} \sum_{\substack{\delta_{j i_{2} \cdots i_{m}}=0, \delta_{q i_{2} \cdots i_{m}}=0}} a_{j i_{2} \cdots i_{m}} x_{i_{2}} \cdots x_{i_{m}} .
\end{aligned}
$$

From Theorem 2.1 in [9], we have $\tau(\mathcal{A}) \leq \min _{i \in N} a_{i \ldots i}$ and

$$
\begin{aligned}
& \left(\left(a_{q \cdots q}-\tau(\mathcal{A})\right)\left(a_{j \cdots j}-\tau(\mathcal{A})\right)-a_{q j \cdots j} a_{j q \cdots q}\right) x_{q}^{m-1} \\
& \quad=\left(a_{j \cdots j}-\tau(\mathcal{A})\right) \sum_{\substack{\delta_{q i_{2} \cdots i_{m}}=0, \delta_{j i_{2} \cdots i_{m}}=0}}\left|a_{q i_{2} \cdots i_{m}}\right| x_{i_{2}} \cdots x_{i_{m}}+\left|a_{q j \cdots j}\right| \sum_{\substack{\delta_{j j_{2} \cdots i_{m}}=0, \delta_{q i_{2} \cdots i_{m}}=0}}\left|a_{j i_{2} \cdots i_{m}}\right| x_{i_{2}} \cdots x_{i_{m}} .
\end{aligned}
$$


Hence,

$$
\begin{aligned}
& \left(\left(a_{q \cdots q}-\tau(\mathcal{A})\right)\left(a_{j \cdots j}-\tau(\mathcal{A})\right)-\left|a_{q j \cdots j}\right|\left|a_{j q \cdots q}\right|\right) x_{q}^{m-1} \\
& \geq\left(a_{j \cdots j}-\tau(\mathcal{A})\right) \sum_{\substack{\delta_{q i_{2} \cdots i_{m}}=0, \delta_{j i_{2} \cdots i_{m}}=0}}\left|a_{q i_{2} \cdots i_{m}}\right| x_{q}^{m-1}+\left|a_{q j \cdots j}\right| \sum_{\substack{\delta_{j i_{2} \cdots i_{m}}=0, \delta_{q i_{2} \cdots i_{m}}=0}}\left|a_{j i_{2} \cdots i_{m}}\right| x_{q}^{m-1} .
\end{aligned}
$$

From $x_{q}>0$, we have

$$
\begin{aligned}
& \left(a_{q \cdots q}-\tau(\mathcal{A})\right)\left(a_{j \cdots j}-\tau(\mathcal{A})\right)-\left|a_{q j \cdots j}\right|\left|a_{j q \cdots q}\right| \\
& \geq\left(a_{j \cdots j}-\tau(\mathcal{A})\right) \sum_{\substack{\delta_{q i_{2} \cdots i_{m}}=0, \delta_{j i_{2} \cdots i_{m}}=0}}\left|a_{q i_{2} \cdots i_{m}}\right|+\left|a_{q j \cdots j}\right| \sum_{\substack{\delta_{j i_{2} \cdots i_{m}}=0, \delta_{q i_{2} \cdots i_{m}}=0}}\left|a_{j i_{2} \cdots i_{m}}\right| \\
& =\left(a_{j \cdots j}-\tau(\mathcal{A})\right) r_{q}^{j}(\mathcal{A})+\left|a_{q j \cdots j}\right| r_{j}^{q}(\mathcal{A}),
\end{aligned}
$$

equivalently,

$$
\left(a_{q \cdots q}-\tau(\mathcal{A})\right)\left(a_{j \cdots j}-\tau(\mathcal{A})\right)-\left(a_{j \cdots j}-\tau(\mathcal{A})\right) r_{q}^{j}(\mathcal{A})-\left|a_{q j \cdots j}\right| r_{j}(\mathcal{A}) \geq 0,
$$

that is,

$$
\tau(\mathcal{A})^{2}-\left(a_{q \cdots q}+a_{j \cdots j}-r_{q}^{j}(\mathcal{A})\right) \tau(\mathcal{A})+a_{q \cdots q} a_{j \cdots j}-a_{j \ldots j} r_{q}^{j}(\mathcal{A})+a_{q j \cdots j} r_{j}(\mathcal{A}) \geq 0
$$

Solving for $\tau(\mathcal{A})$ gives

$$
\tau(\mathcal{A}) \leq \frac{1}{2}\left\{a_{q \cdots q}+a_{j \cdots j}-r_{q}^{j}(\mathcal{A})-\left[\left(a_{q \cdots q}-a_{j \cdots j}-r_{q}^{j}(\mathcal{A})\right)^{2}-4 a_{q j \cdots j} r_{j}(\mathcal{A})\right]^{\frac{1}{2}}\right\}=L_{q j}(\mathcal{A})
$$

For the arbitrariness of $j$, we have $\tau(\mathcal{A}) \leq \min _{j \neq q} L_{q j}(\mathcal{A})$. Furthermore, we have

$$
\tau(\mathcal{A}) \leq \max _{i \in N} \min _{j \neq i} L_{i j}(\mathcal{A})
$$

(II) Let $x_{p}=\max \left\{x_{i}: i \in N\right\}$. Similar to (I), we have

$$
\tau(\mathcal{A}) \geq \min _{i \in N} \max _{j \neq i} L_{i j}(\mathcal{A})
$$

The conclusion follows from (I) and (II).

Similar to the proof of Theorem 3.6 in [2], we can extend the results of Theorem 6 to a more general case.

Theorem 7 Let $\mathcal{A}=\left(a_{i_{1} \cdots i_{m}}\right) \in \mathbb{R}^{[m, n]}$ be an $\mathcal{M}$-tensor. Then

$$
\min _{i \in N} \max _{j \neq i} L_{i j}(\mathcal{A}) \leq \tau(\mathcal{A}) \leq \max _{i \in N} \min _{j \neq i} L_{i j}(\mathcal{A})
$$

By Theorems 3, 6 and 7 in [13], the following comparison theorem is obtained easily. 
Theorem 8 Let $\mathcal{A}=\left(a_{i_{1} \cdots i_{m}}\right) \in \mathbb{R}^{[m, n]}$ be an $\mathcal{M}$-tensor, $S$ be a nonempty proper subset of $N$, $\bar{S}$ be the complement of $S$ in $N$. Then

$$
\begin{aligned}
\min _{i \in N} R_{i}(\mathcal{A}) & \leq \min _{j \neq i} L_{i j}(\mathcal{A}) \leq \min \left\{\min _{i \in S} \max _{j \in \bar{S}} L_{i j}(\mathcal{A}), \min _{i \in \bar{S}} \max _{j \in S} L_{i j}(\mathcal{A})\right\} \leq \min _{i \in N} \max _{j \neq i} L_{i j}(\mathcal{A}) \\
& \leq \max _{i \in N} \min _{j \neq i} L_{i j}(\mathcal{A}) \leq \max \left\{\max _{i \in S} \min _{j \in \bar{S}} L_{i j}(\mathcal{A}), \max _{i \in \bar{S}} \min _{j \in S} L_{i j}(\mathcal{A})\right\}
\end{aligned}
$$

where $R_{i}(\mathcal{A})=\sum_{i_{2}, \ldots, i_{m} \in N} a_{i i_{2} \cdots i_{m}}$.

Remark 2 Theorem 8 shows that the bounds in Theorem 7 are shaper than those in Theorem 3, Theorem 2.1 of [9] and Theorem 4 of [13] without considering the selection of $S$, which is also the advantage of our results.

\section{Numerical examples}

In this section, two numerical examples are given to verify the theoretical results.

Example 1 Let $\mathcal{A}=\left(a_{i j k}\right) \in \mathbb{R}^{[3,4]}$ be an irreducible $\mathcal{M}$-tensor with elements defined as follows:

$$
\begin{aligned}
& \mathcal{A}(:,:, 1)=\left(\begin{array}{llll}
62 & -3 & -4 & -2 \\
-4 & -2 & -2 & -1 \\
-3 & -1 & -3 & -3 \\
-3 & -3 & -2 & -2
\end{array}\right), \quad \mathcal{A}(:,:, 2)=\left(\begin{array}{cccc}
0 & -4 & -3 & -3 \\
-1 & 28 & -2 & -2 \\
-1 & -2 & -2 & -4 \\
-2 & -2 & -3 & -1
\end{array}\right), \\
& \mathcal{A}(:,:, 3)=\left(\begin{array}{llll}
-2 & -1 & -2 & -1 \\
-1 & -1 & -1 & -2 \\
-2 & -4 & 63 & -4 \\
-4 & -4 & -2 & -2
\end{array}\right), \quad \mathcal{A}(:,,, 4)=\left(\begin{array}{cccc}
-4 & -2 & -2 & -1 \\
-1 & -2 & -3 & -1 \\
-2 & -3 & -3 & -2 \\
-2 & -2 & -4 & 61
\end{array}\right) .
\end{aligned}
$$

By Theorem 2.1 in [9], we have

$$
2=\min _{i \in N} R_{i}(\mathcal{A}) \leq \tau(\mathcal{A}) \leq \min \left\{\max _{i \in N} R_{i}(\mathcal{A}), \min _{i \in N} a_{i \cdots i}\right\}=28 .
$$

By Theorem 4 in [13], we have

$$
\tau(\mathcal{A}) \geq \min _{j \neq i} L_{i j}(\mathcal{A})=2.3521 .
$$

By Theorem 3, we have

$$
\begin{array}{ll}
\text { if } S=\{1\}, \bar{S}=\{2,3,4\}, & 3.6685 \leq \tau(\mathcal{A}) \leq 24.2948 ; \\
\text { if } S=\{2\}, \bar{S}=\{1,3,4\}, & 3.6685 \leq \tau(\mathcal{A}) \leq 19.7199 ; \\
\text { if } S=\{3\}, \bar{S}=\{1,2,4\}, & 2.3569 \leq \tau(\mathcal{A}) \leq 27.7850 ; \\
\text { if } S=\{4\}, \bar{S}=\{1,2,3\}, & 2.3521 \leq \tau(\mathcal{A}) \leq 27.8536 ; \\
\text { if } S=\{1,2\}, \bar{S}=\{3,4\}, & 2.3569 \leq \tau(\mathcal{A}) \leq 27.7850 ; \\
\text { if } S=\{1,3\}, \bar{S}=\{2,4\}, & 3.6685 \leq \tau(\mathcal{A}) \leq 23.0477 ;
\end{array}
$$




$$
\text { if } S=\{1,4\}, \bar{S}=\{2,3\}, \quad 3.6685 \leq \tau(\mathcal{A}) \leq 23.9488
$$

By Theorem 7, we have

$$
3.6685 \leq \tau(\mathcal{A}) \leq 19.7199 .
$$

In fact, $\tau(\mathcal{A})=14.4049$. Hence, this example verifies Theorem 8 and Remark 2 , that is, the bounds in Theorem 7 are sharper than those in Theorem 3, Theorem 2.1 of [9] and Theorem 4 of [13] without considering the selection of $S$.

Example 2 Let $\mathcal{A}=\left(a_{i j k l}\right) \in \mathbb{R}^{[4,2]}$ be an $\mathcal{M}$-tensor with elements defined as follows:

$$
a_{1111}=6, \quad a_{1222}=-1, \quad a_{2111}=-2, \quad a_{2222}=5,
$$

other $a_{i j k l}=0$. By Theorem 7, we have

$$
4 \leq \tau(\mathcal{A}) \leq 4
$$

In fact, $\tau(\mathcal{A})=4$.

\section{Conclusions}

In this paper, we give a new eigenvalue inclusion set for tensors and prove that this set is tighter than those in $[1,2]$. As an application, we obtain new lower and upper bounds for the minimum eigenvalue of $\mathcal{M}$-tensors and prove that the new bounds are sharper than those in $[2,9,13]$. Compared with the results in [2], the advantage of our results is that, without considering the selection of $S$, we can obtain a tighter eigenvalue localization set for tensors and sharper bounds for the minimum eigenvalue of $\mathcal{M}$-tensors.

Competing interests

The authors declare that they have no competing interests.

Authors' contributions

All authors contributed equally to this work. All authors read and approved the final manuscript.

\section{Acknowledgements}

This work is supported by the National Natural Science Foundation of China (Nos. 11361074, 11501141), the Foundation of Guizhou Science and Technology Department (Grant No. [2015]2073) and the Natural Science Programs of Education Department of Guizhou Province (Grant No. [2016]066).

Received: 15 January 2017 Accepted: 27 February 2017 Published online: 09 March 2017

\section{References}

1. Li, CQ, Chen, Z, Li, YT: A new eigenvalue inclusion set for tensors and its applications. Linear Algebra Appl. 481, 36-53 (2015)

2. Huang, ZG, Wang, LG, Xu, Z, Cui, JJ: A new S-type eigenvalue inclusion set for tensors and its applications. J. Inequal. Appl. 2016, 254 (2016)

3. Chang, KQ, Zhang, T, Pearson, K: Perron-Frobenius theorem for nonnegative tensors. Commun. Math. Sci. 6, 507-520 (2008)

4. Qi, LQ: Eigenvalues of a real supersymmetric tensor. J. Symb. Comput. 40, 1302-1324 (2005)

5. Lim, LH: Singular values and eigenvalues of tensors: a variational approach. In: Proceedings of the IEEE International Workshop on Computational Advances in Multi-Sensor Adaptive Processing. CAMSAP, vol. 05, pp. 129-132 (2005)

6. Yang, YN, Yang, QZ: Further results for Perron-Frobenius theorem for nonnegative tensors. SIAM J. Matrix Anal. Appl. 31, 2517-2530 (2010)

7. Ding, WY, Qi, LQ, Wei, YM: $\mathcal{M}$-tensors and nonsingular $\mathcal{M}$-tensors. Linear Algebra Appl. 439, 3264-3278 (2013)

8. Zhang, LP, Qi, LQ, Zhou, GL: $\mathcal{M}$-tensors and some applications. SIAM J. Matrix Anal. Appl. 35, 437-452 (2014)

9. He, J, Huang, TZ: Inequalities for $\mathcal{M}$-tensors. J. Inequal. Appl. 2014, 114 (2014) 
10. Li, CQ, Li, YT, Kong, X: New eigenvalue inclusion sets for tensors. Numer. Linear Algebra Appl. 21, 39-50 (2014)

11. Li, CQ, Li, YT: An eigenvalue localization set for tensor with applications to determine the positive (semi-)definiteness of tensors. Linear Multilinear Algebra 64(4), 587-601 (2016)

12. Li, CQ, Jiao, AQ, Li, YT: An S-type eigenvalue location set for tensors. Linear Algebra Appl. 493, 469-483 (2016)

13. Zhao, JX, Sang, CL: Two new lower bounds for the minimum eigenvalue of $\mathcal{M}$-tensors. J. Inequal. Appl. 2016, 268 (2016)

14. He, J: Bounds for the largest eigenvalue of nonnegative tensors. J. Comput. Anal. Appl. 20(7), 1290-1301 (2016)

Submit your manuscript to a SpringerOpen ${ }^{\circ}$ journal and benefit from:

$\rightarrow$ Convenient online submission

Rigorous peer review

- Immediate publication on acceptance

- Open access: articles freely available online

- High visibility within the field

- Retaining the copyright to your article 